Editor's Note: These short reviews of a recent paper in the Journal, written exclusively by graduate students or postdoctoral fellows, are intended to mimic the journal clubs that exist in your own departments or institutions. For more information on the format and purpose of the Journal Club, please see http://www.jneurosci.org/misc/ifa_features.shtml.

\title{
Stimulation-Induced Dyskinesias Inform Basal Ganglia Models and the Mechanisms of Deep Brain Stimulation
}

\author{
Jason T. Moyer and Shabbar F. Danish \\ Review of Boulet et al. (http://www.jneurosci.org/cgi/content/full/26/42/10768)
}

High-frequency (or deep brain) stimulation (HFS) of the subthalamic nucleus (STN) represents an effective treatment for intermediate to late-stage Parkinson's disease (PD). The success of HFS for treatment of PD has accelerated research into its use for a number of other disorders, ranging from essential tremor to depression. Despite its success, it is still not clear how HFS changes activity in the brain to achieve its clinical effect. Recent work by Boulet et al. (2006) in The Journal of Neuroscience supports previous studies finding that HFS activates the target structure. Further, it provides new evidence suggesting that dyskinesias (a common side-effect of long-term dopaminereplacement therapy for PD as well as high-intensity HFS) are correlated with increases in glutamate in the substantia nigra pars reticulata $(\mathrm{SNr})$. This work has implications for both understanding of the mechanisms of HFS as well as understanding of the relationships between neural structures in the basal ganglia (BG).

HFS has been successfully applied to a number of target neural structures. For PD, the STN seems to be the most effective target, because it improves all symptoms (tremor, slowed and reduced movements, and rigidity), and permits medication to be reduced in many cases. This is an important benefit of HFS, in that long-term (5-10 years) drug therapy (using levo-

Received Dec. 12, 2006; revised Jan. 3, 2007; accepted Jan. 4, 2007. Correspondence should be addressed to Jason Moyer, University of Pennsylvania, Department of Bioengineering, 210 South 33rd Street, 240 Skirkanich Hall, Philadelphia, PA 19104. E-mail: jtmoyer@seas.upenn.edu. DOI:10.1523/JNEUROSCI.5359-06.2007

Copyright $\odot$ 2007 Society for Neuroscience $\quad$ 0270-6474/07/271799-02\$15.00/0 dopa, which is converted into dopamine in the brain) can lead to severe dyskinesias that are almost as disabling as PD itself. Dyskinesias are repetitive, involuntary movements such as wrist or ankle flexion and extension or hand clenching. Dyskinesias can also be induced with highintensity HFS.

Before the development of HFS, lesioning was the primary surgical treatment for PD. Based on the similar clinical efficacy of the two procedures, it was assumed that HFS constituted a functional lesion of the target structure. Several studies appeared to support this concept, which primarily consisted of recordings within the target structure during and immediately after HFS. However, subsequent electrophysiology recordings, microdialysis, and functional imaging of downstream structures suggested that the target structure is actually activated (for review, see Moyer et al., 2007). HFS may decouple axonal activity from somatic activity, with somatic activity inhibited by HFS, but axonal output increased (McIntyre et al., 2004), which could explain these disparate findings.

Boulet et al. (2006) used 6-OHDA to model PD in rats by selectively lesioning the substantia nigra pars compacta $(\mathrm{SNc})$, which represents the dopaminergic input to the dorsal striatum and BG. They simulated HFS by stimulating at $130 \mathrm{~Hz}$ via implanted microelectrodes in the STN in both 6-OHDA-lesioned rats and a control group with an intact SNc. They performed microdialysis in the $\mathrm{SNr}$ of both groups, sampling every $15 \mathrm{~min}$ over a period of $5 \mathrm{~h}$ before, during, and after HFS in awake animals.

Boulet et al. (2006) describe four cate- gories of dyskinesias that were observed in the 6-OHDA-lesioned group and in the control group as the amount of stimulating current was increased [Boulet et al. (2006), their Fig. 2 (http://www.jneurosci. org/cgi/content/full/26/42/10768/F2)]. The authors focused their attention on forelimb dyskinesias, presumably because these most nearly approximate the hand and foot dyskinesias, which are most disabling in PD patients. Thus, it is unclear whether the results also would apply to other types of dyskinesia.

The primary findings of Boulet et al. (2006) are as follows: (1) HFS stimulation at a threshold intensity for the induction of forelimb dyskinesia (stimulation amplitude $\mathrm{I}_{1}$ ) resulted in increased extracellular SNr glutamate during and after HFS in both intact and 6-OHDA-lesioned rats [Boulet et al. (2006), their Fig. 3 (http:// www.jneurosci.org/cgi/content/full/26/ 42/10768/F3)]; (2) HFS stimulation at half the threshold intensity for induction of forelimb dyskinesia (stimulation amplitude $\mathrm{I}_{2}$ ) did not result in increased glutamate levels in the SNr of either intact or 6-OHDA-lesioned rats [Boulet et al. (2006), their Fig. 4 (http://www.jneurosci. org/cgi/content/full/26/42/10768/F4)]; (3) injecting the glutamate receptor (GluR) antagonist kynurenic acid into the SNr during $\mathrm{I}_{1}$ stimulation prevented forelimb dyskinesias [Boulet et al. (2006), their Fig. 5 (http://www.jneurosci.org/ cgi/content/full/26/42/10768/F5)]; and (4) injecting GluR agonists AMPA and NMDA into the $\mathrm{SNr}$ during $\mathrm{I}_{2}$ stimulation induced forelimb dyskinesia. Injecting AMPA and NMDA without stimulation was not sufficient to induce forelimb dyskinesia [Boulet et al. (2006), their Fig. 5 
(http://www.jneurosci.org/cgi/content/ full/26/42/10768/F5)]. Based on this evidence, the authors contend that STN HFS-induced forelimb dyskinesias are mediated by glutamate in the SNr.

Given that the glutamate levels in the SNr appear to derive primarily from STN activity (Rosales et al., 1997), the increases in extracellular glutamate during and after HFS support the concept that HFS activates the target structure. However, it cannot be deduced from the work of Boulet et al. (2006) whether this increase is necessary for clinical improvement, because data were not included regarding the physical state of the rats (such as increased number of nondyskinetic movements during HFS). Boulet et al. (2006) also found that GABA levels could be increased by HFS, and that AMPA and NMDA injections into the $\mathrm{SNr}$ alone are not sufficient to induce dyskinesias. Especially when considered with a previous microdialysis study trying levodopainduced dyskinesias to glutamate increases in both the striatum and the substantia nigra (Robelet et al., 2004), this illustrates that HFS affects not only the target structure and its immediate downstream targets, but somehow alters activity throughout the BG in a more complicated way.

If HFS activates glutamate-releasing STN projections, this has interesting implications for understanding of the BG. In the classical model of Parkinson's disease (Albin et al., 1989; DeLong, 1990), loss of DA innervation of the striatum increases activity in the indirect, movementsuppressing pathway, and reduces activity in the direct, movement-facilitating pathway (Fig. 1). Thus, according to this model, the Parkinsonian STN should be hyperactive as a result of reduced GPe (external segment of the globus pallidus) inhibitory transmission. The finding by Boulet et al. (2006) that basal glutamate levels are increased in the SNr of 6-OHDAlesioned rats [Boulet et al. (2006), their Table 1 (http://www.jneurosci.org/cgi/content/ full/26/42/10768/T1)] supports this prediction. However, the finding by Boulet et al. (2006) that increased glutamate in the $\mathrm{SNr}$ increases movements (albeit nonphysiological ones) contradicts this model's prediction that STN hyperactivity should reduce movements (as in Parkinson's disease).

One of the shortcomings of the microdialysis technique is its inability to capture brief, small-scale changes in neural behavior. For example, it has been suggested that HFS might alleviate symptoms by replacing STN bursting activity with regular

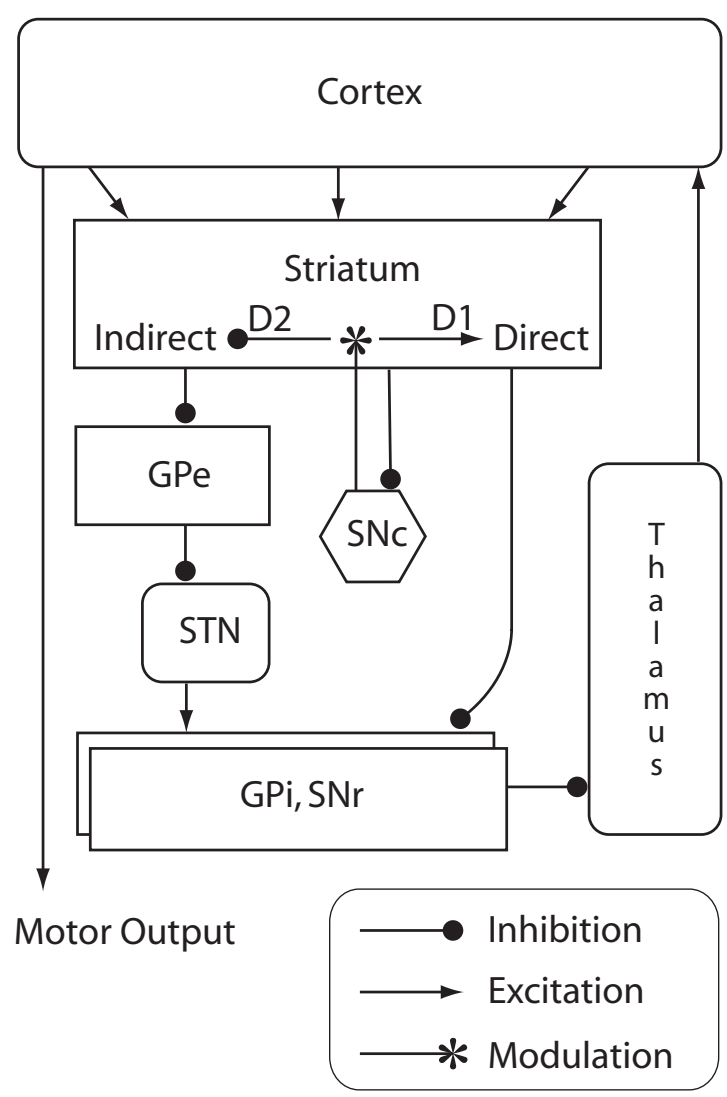

Figure 1. Classical model of the basal ganglia (Albin et al., 1989; DeLong, 1990). Death of dopaminergic neurons in the SNc reduces inhibition of the indirect pathway via dopamine $D_{2}$ receptors and reduces activation of the direct pathway via dopamine $D_{1}$ receptors. Accordingly, the net effect of dopamine loss, as in Parkinson's disease, is increased inhibition of thalamocortical activity and impaired movement. This model predicts that basal activity of the STN should be increased in Parkinson's disease. GPe, External segment of the globus pallidus; GPi, internal segment of the globus pallidus.

spiking (for review, see McIntyre et al., 2004); this possibility cannot be investigated using microdialysis. Also, several groups have described oscillatory activity throughout the BG that appears to be very important for both BG and HFS function (for review, see Moyer et al., 2007). Again, techniques with higher time resolution than microdialysis will need to be used to investigate how oscillatory activity governs BG function and what this might mean for the mechanisms of HFS.

Future studies will need to determine whether other target structures are also activated by HFS, and if so, whether this activation is required for clinical efficacy. They will also have to explore how HFS of the STN affects BG dynamics. The results of these experiments should facilitate the use of HFS, and hopefully suggest ways in which HFS can be improved and expanded to treat other diseases.

\section{References}

Albin RL, Young AB, Penney JB (1989) The functional anatomy of basal ganglia disorders. Trends Neurosci 12:366-375.

Boulet S, Lacombe E, Carcenac C, Feuerstein C,
Sgambato-Faure V, Poupard A, Savasta M (2006) Subthalamic stimulation-induced forelimb dyskinesias are linked to an increase in glutamate levels in the substantia nigra pars reticulata. J Neurosci 26:10768-10776.

DeLong MR (1990) Primate models of movement disorders of basal ganglia origin. Trends Neurosci 13:281-285.

McIntyre CC, Savasta M, Kerkerian-Le Goff L, Vitek JL (2004) Uncovering the mechanism(s) of action of deep brain stimulation: activation, inhibition, or both. Clin Neurophysiol 115:1239-1248.

Moyer JT, Danish SF, Finkel LH (2007) Deep brain stimulation: anatomical, physiological, and computational mechanisms. In: Deep brain stimulation for Parkinson's disease: a comprehensive text (Baltuch GH, Stern MB, eds), pp 17-40. New York: Informa Healthcare.

Robelet S, Melon C, Salin P, Kerkerian-Le Goff L (2004) Chronic L-DOPA treatment increases extracellular glutamate levels and GLT1 expression in the basal ganglia in a rat model of Parkinson's disease. Eur J Neurosci 20:1255-1266.

Rosales MG, Martinez-Fong D, Morales R, Nunez A, Flores G, Gongora-Alfaro JL, Floran B, Aceves J (1997) Reciprocal interaction between glutamate and dopamine in the pars reticulata of the rat substantia nigra: a microdialysis study. Neuroscience 80:803-810. 